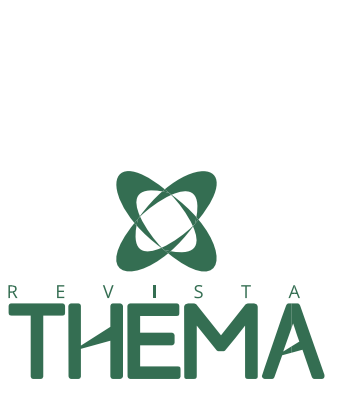

\title{
ENGENHARIAS \\ Construção de Sistema embarcado para controle sem fio de tensão alternada em experimentos de física
}

\section{Design of embedded system for wireless control of AC voltage in Physics experiments}

\author{
Renata Imaculada Soares Pereira1; Sandro César Silveira Jucá2; Josué Batista \\ Mota $^{2}$
}

\section{RESUMO}

Este artigo descreve a construção, a programação e a implementação de um sistema didático composto de softwares educacionais livres e de um sistema embarcado de controle de tensão senoidal alternada para práticas de eletricidade em laboratórios de Física. Nesse sentido, esse trabalho propõe um software educacional desenvolvido e aplicado em disciplinas de Eletricidade de cursos técnicos e superiores para o controle digital, de forma sem fio via Bluetooth, para variação da tensão alternada da rede elétrica utilizando smartphones ou tablets Android, tornando o experimento mais interativo e lúdico. A aplicação de softwares educacionais em experimentos permite também a verificação, em tempo real, da influência da alteração de programas nos fenômenos físicos, o que estimula o desenvolvimento do raciocínio lógico e, consequentemente, da autonomia dos alunos, à medida que podem levantar hipóteses, fazer interferências na programação e tirar conclusões a partir dos resultados obtidos na prática.

Palavras-chave: Android; Bluetooth; Eletricidade; Física experimental; Software educacional

\begin{abstract}
This paper describes the construction, programming and implementation of an embedded system based on free educational software and an embedded control of sinusoidal alternating voltage (AC) for electricity practices in Physics laboratory. Thus, this work presents educational software developed and applied in subjects of Electricity of technical and undergraduate courses for the digital control, wirelessly via Bluetooth, to change the $A C$ voltage of the grid using Android smartphones or tablets, making the experiment more interactive and playful. The application of educational software in experiments also allows to verify, in real time, the influence of programs change on the physical phenomena and stimulates the logical reasoning development and consequently the autonomy of the students, to the measure that can raise hypotheses, make interferences in the programming and take off conclusions from the practical results obtained.
\end{abstract}

Keywords: Android; Bluetooth; Educational software; Electricity; Experimental Physics.

\footnotetext{
${ }^{1}$ UFC - Universidade Federal do Ceará, Fortaleza/CE - Brasil.

2 IFCE - Instituto Federal de Educação, Ciência e Tecnologia do Ceará, Maracanaú/CE - Brasil.
} 


\section{INTRODUÇÃO}

Atualmente existem diversos equipamentos comerciais (sistemas proprietários) projetados para ensino de física experimental, de elevado custo, devido também às taxas de importação, transporte e impostos (OLIVEIRA et al., 2011). Estas plataformas são consideradas "caixas pretas" construídas para uso nos laboratórios didáticos de ensino de física (ROCHA et al., 2014). Por outro lado, é comum, no processo de ensino-aprendizagem, o desenvolvimento de experimentos de física em baixo custo, utilizando sistemas embarcados ou microcontrolados e programados com software livre (SOUZA, 2011; CAVALCANTE et al., 2011; CAVALCANTE, 2013). Neste momento histórico da sociedade da informação, essas novas tecnologias mostram que, quando utilizadas adequadamente, auxiliam na construção do conhecimento, tornando o processo de ensino-aprendizagem mais estimulante e mais eficiente na interação com o mundo real. Dessa forma, sistemas microcontrolados são ferramentas muito úteis para o desenvolvimento de práticas voltadas ao ensino de física experimental e, juntamente com os softwares livres, podem contribuir de forma eficaz no processo de ensinoaprendizagem (SOUZA, 2011).

Nesse sentido, é recomendado, nos Parâmetros Curriculares Nacionais, desenvolver ferramentas educacionais consistentes com novos métodos de ensino-aprendizagem compatíveis com tecnologias atuais (BRASIL, 2000). Além disso, a inserção criteriosa de recursos tecnológicos no ambiente de aprendizado através de atividades práticas de laboratório pode ajudar a despertar vocações para as carreiras tecnológicas do ensino superior (ROCHA et al., 2014).

Existem diversas ferramentas que auxiliam no ambiente educacional, como por exemplo, o WhatsApp, um instrumento que proporciona um espaço administrativo entre os estudantes e professores, no sentido do compartilhamento das disciplinas (DE BONA, 2016). Outro meio didático proposto, foi a construção de um equipamento que realiza uma atividade experimental para visualizar o fenômeno da difração e interferência da luz, afim de levar uma experiência interativa para os estudantes (DARROZ, 2016). Neste contexto, este trabalho tem o objetivo de descrever a construção, a programação e a implementação de um sistema didático composto de um software educacional livre e de um sistema embarcado de controle de tensão senoidal alternada para práticas de eletricidade em laboratórios de física.

\section{CIRCUITO MICROCONTROLADO}

A comunicação do circuito microcontrolado com o aplicativo Android pode ser realizada também utilizando protocolos de comunicação sem fio, como Bluetooth e WiFi. Neste experimento, a forma de comunicação sem fio é realizada com o protocolo Bluetooth. Na transmissão Bluetooth, a comunicação é efetuada aplicando-se uma técnica de espalhamento espectral com saltos de frequência, denominada de FHSS (Frequency Hopping Spread Spectrum).

Desta forma, a frequência nunca é a mesma, minimizando as interferências e possibilitando a existência de outras redes Bluetooth na mesma área geográfica (MULLER, 2011). Os requisitos principais que nortearam o desenvolvimento do Bluetooth foram o baixo consumo, o baixo custo devido à produção em massa dos chips, o alcance de até 100 metros (no caso do módulo utilizado neste trabalho, pode-se comunicar a $30 \mathrm{~m}$ ) e transmissão de voz e de dados. A Figura 1a ilustra a conexão do circuito microcontrolado desenvolvido com o módulo Bluetooth utilizando quatro fios e a Figura 1b ilustra a montagem real do circuito microcontrolado conectado ao modem Bluetooth. 


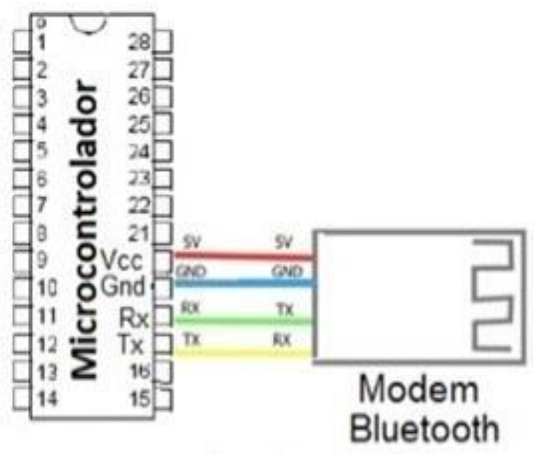

Figura 1a: Ilustração da conexão do circuito microcontrolado desenvolvido ao modem Bluetooth.

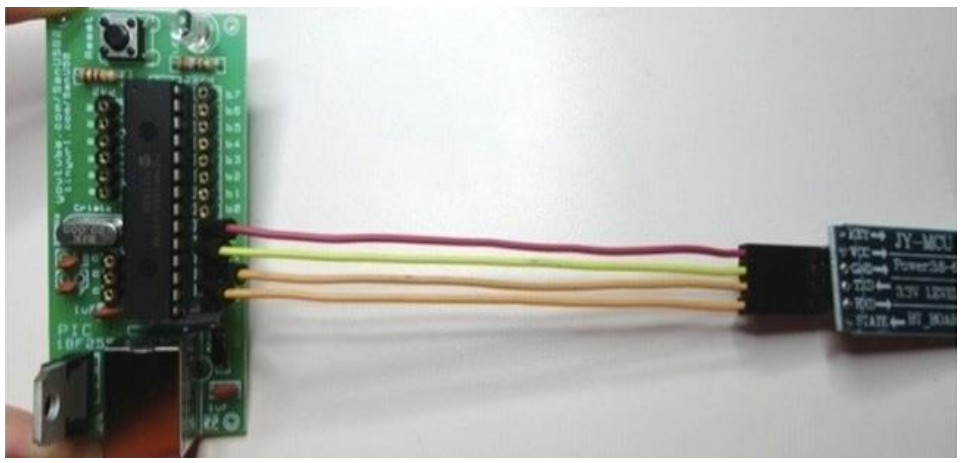

Figura 1b: Montagem real do circuito microcontrolado conectado ao modem Bluetooth.

Neste experimento, foi desenvolvido um programa em linguagem C (firmware), que deve ser gravado no circuito microcontrolado para que este possa receber as informações dos dispositivos Android via Bluetooth, como smartphones ou tablets, e variar a tensão alternada eficaz $\left(\mathrm{V}_{\mathrm{CA}}\right)$ de uma carga, que no caso desse trabalho proposto é uma lâmpada incandescente, através do circuito de controle digital. O processo de comunicação do dispositivo Android até uma carga alimentada com tensão alternada é ilustrado na Figura 2. O compilador livre utilizado para o firmware do microcontrolador foi o $\mathrm{C} 18$ juntamente com o ambiente multiplataforma MPLABX. Para possibilitar maior difusão dos softwares educacionais propostos, foi desenvolvido também um programa (sketch) para comunicação com a plataforma Arduino que pode ser acessado diretamente na tela do software educacional aplicativo, ilustrado na Figura 3.

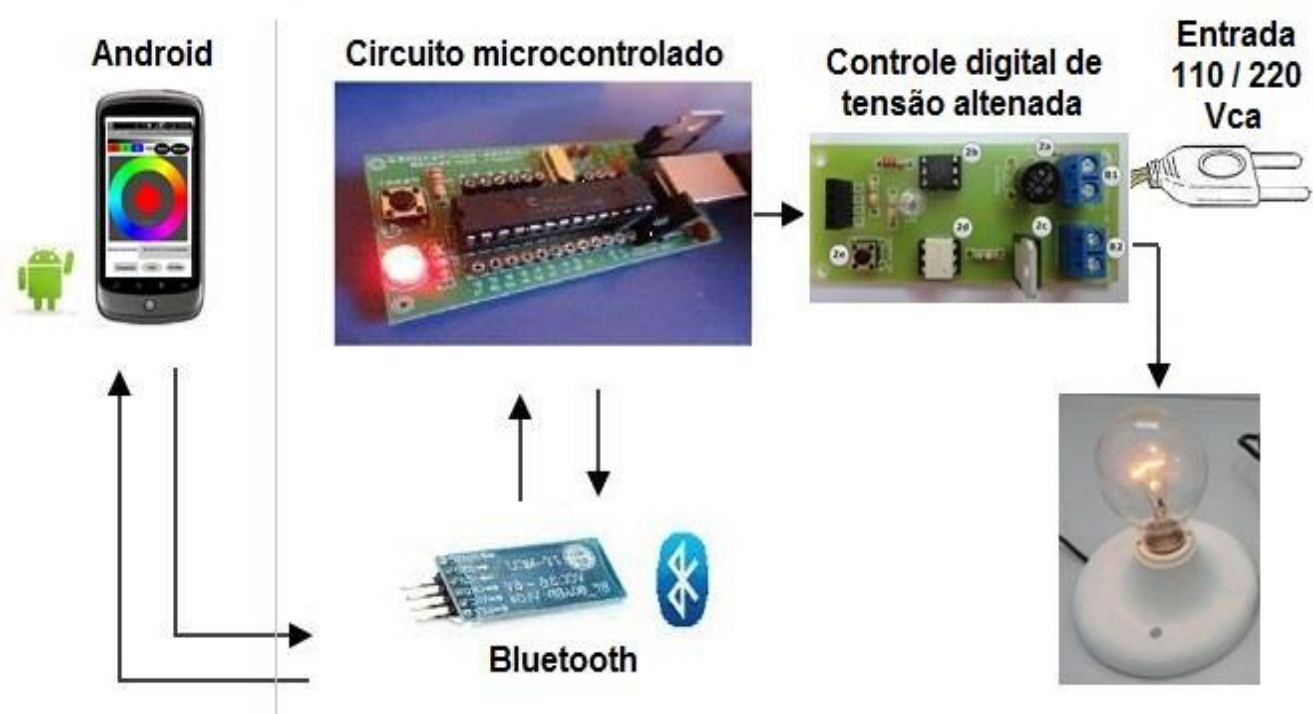

Figura 2: Ilustração do processo de comunicação Bluetooth do dispositivo Android com o circuito microcontrolado para controle digital de tensão senoidal alternada RGB. 


\section{APLICATIVO DESENVOLVIDO PARA DISPOSITIVOS ANDROID}

O presente tópico descreve o funcionamento do software educacional aplicativo denominado BT4SanUSB, desenvolvido e disponibilizado de forma gratuita para dispositivos Android no ambiente Google Play. Este aplicativo desenvolvido para Android permite comandar a variação de tensão alternada via Bluetooth utilizando o disco gradual do aplicativo ou através dos botões Liga e Desliga. Na Figura 3 são mostradas telas do software educacional livre aplicado em física experimental para composição real e virtual de cores, onde a cor virtual é formada pela composição RGB de cores gerada pela tela do aplicativo e a cor real é formada pela composição gerada pela intensidade de brilho de Leds ou lâmpadas incandescentes de cor vermelha (red), verde (green) e azul (blue).
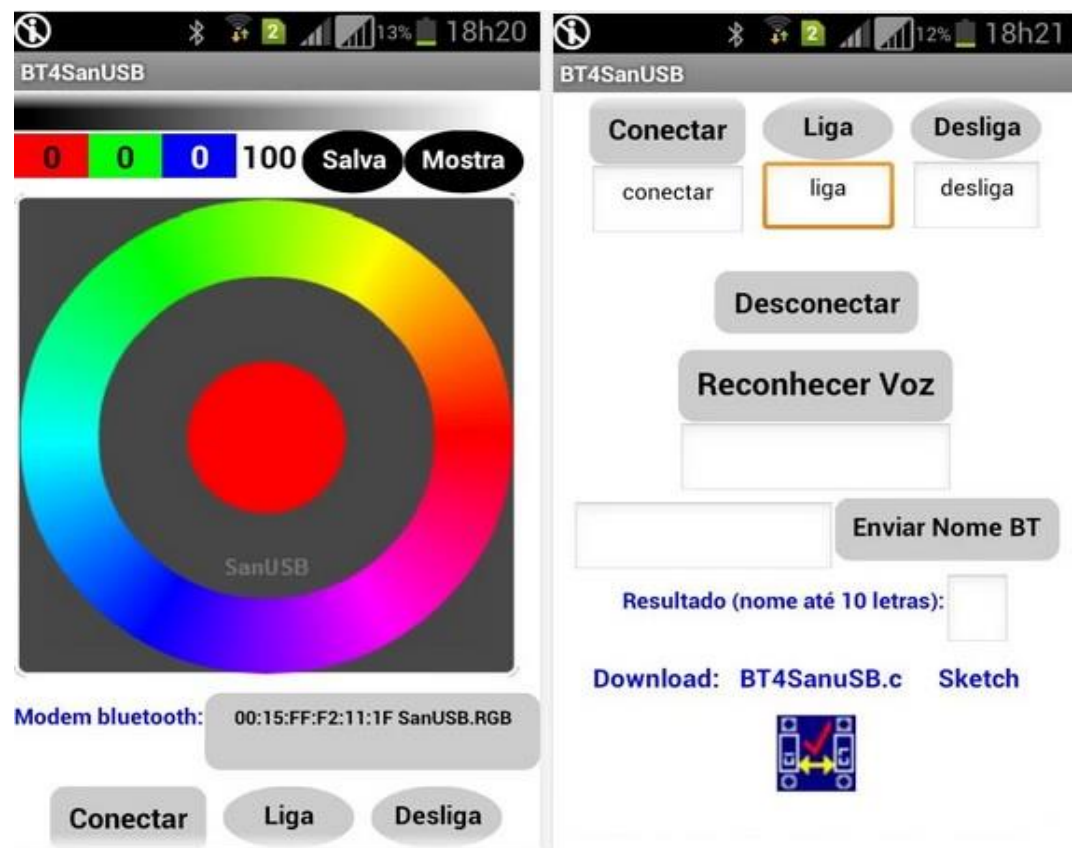

Figura 3: Telas do software educacional aplicativo BT4SanUSB para controle de emissão de cores via Bluetooth.

Este aplicativo realiza também, via Bluetooth, o envio para o microcontrolador dos parâmetros de modulação por largura de pulso, conhecida também como PWM (pulse width modulation) e pode, por exemplo, realizar a mudança real de intensidade de brilho de uma lâmpada incandescente. Entre outras características, o aplicativo permite que uma determinada configuração PWM de um experimento seja armazenada na memória permanente do aplicativo, pressionando o botão "Salva". Este aplicativo possibilita também que o usuário modifique o nome do dispositivo Bluetooth diretamente do dispositivo Android, inserindo o nome na caixa de texto e pressionando o botão "Enviar Nome BT" ao lado.

Além disso, os comandos de voz armazenados na memória permanente do dispositivo Android permitem também corrigir falhas no software de reconhecimento para um determinado comando, pois embora a resposta encontrada pelo software de reconhecimento de voz do Android seja diferente da pronunciada, esta resposta é armazenada mesmo assim e quando este comando de voz é pronunciado novamente, há uma comparação com a palavra armazenada e no caso de palavras iguais, ocorre o reconhecimento e a atuação. 


\section{CIRCUITO DIGITAL DE CONTROLE DE TENSÃO SENOIDAL ALTERNADA}

No intuito de realizar o controle digital do disparo de um TRIAC (Triode for Alternating Current), que é um dispositivo semicondutor utilizado para chavear tensão senoidal tanto no semiciclo positivo quanto no semiciclo negativo da tensão da rede elétrica. Nesse caso, para decidir o momento, em cada semicírculo, de disparo do TRIAC, indicado na Figura 4 por 2c, é necessário identificar inicialmente a passagem pelo zero da tensão da rede (zero-crossing). A detecção de cruzamento pelo zero da tensão senoidal da rede é realizada utilizando inicialmente um circuito foto-acoplador, indicado por $2 \mathrm{~b}$ na Figura 4, na saída de uma ponte de diodos (2a), que é responsável por rebater todos os semicírculos negativos de tensão senoidal para o semicírculo positivo.

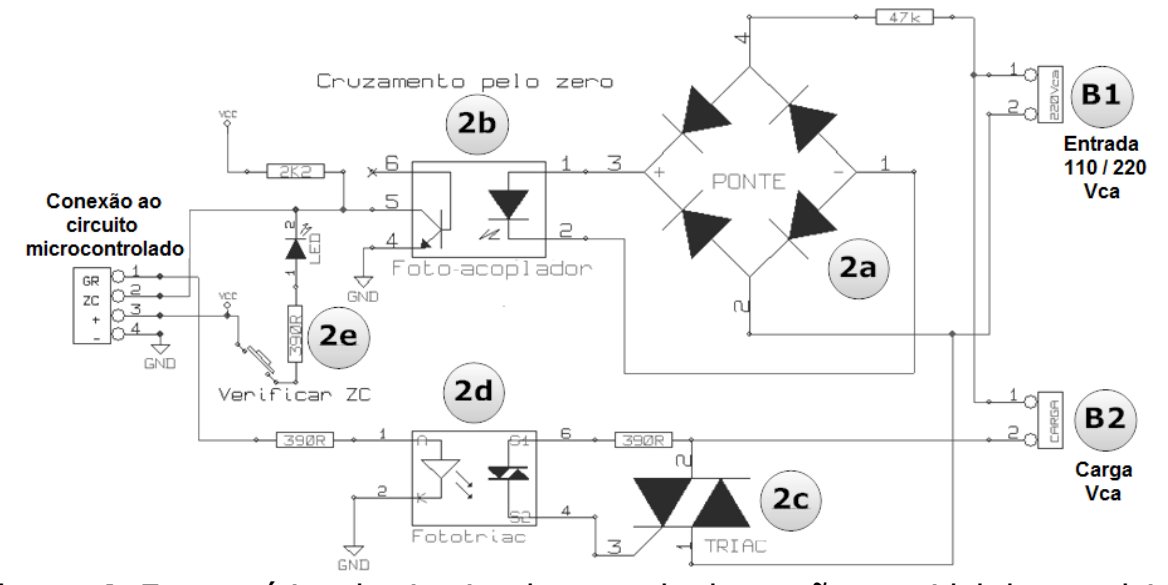

Figura 4: Esquemático do circuito de controle de tensão senoidal desenvolvido.

A forma de identificação da passagem pelo zero da tensão da rede é percebida pelo circuito microcontrolado através do pino de interrupção externa do microcontrolador.

O foto-acoplador é composto internamente por um LED e um foto transistor, utilizado para isolar fisicamente os sinais digitais e proteger os circuitos contra sobretensões. Por outro lado, o fototriac MOC3021, indicado por 2d, é um dispositivo óptico isolador de tensão alternada, sendo necessário para chavear o TRIAC BT08 (2c), tanto no semiciclo negativo quanto no semiciclo positivo da tensão senoidal.

A Figura 5 mostra a foto do circuito de detecção de passagem pelo zero da rede com foto-acoplador e o circuito de atuação do TRIAC foto-acoplado. Foi inserido também na placa desenvolvida um botão (2e) que permite verificar a conexão à rede elétrica. A tensão da rede elétrica ( $220 \mathrm{Vca}$ ) é inserida no borne B1 e a carga é conectada ao borne B2.

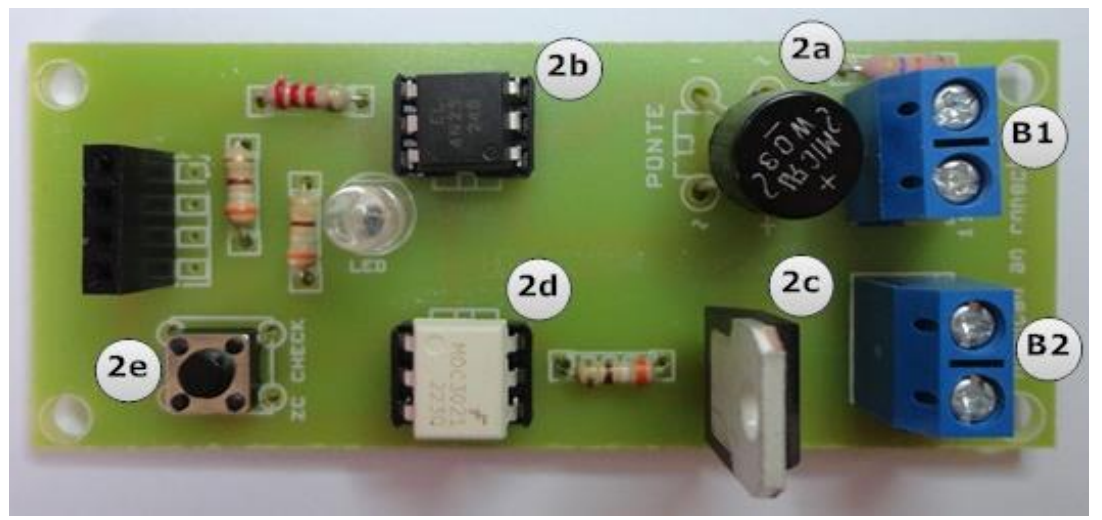

Figura 5: Circuito de controle de tensão senoidal alternada 
A Placa desenvolvida de circuito impresso para o controle digital de tensão senoidal alternada pode ser visto na Figura 6.

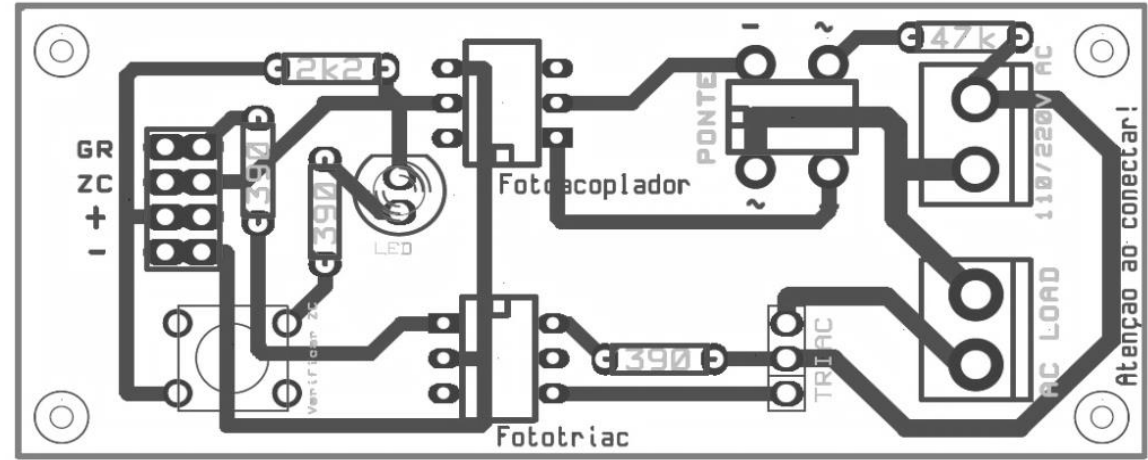

Figura 6: Placa desenvolvida de circuito impresso para o controle digital de tensão senoidal alternada.

Neste controle digital de tensão senoidal, em um dado momento de cada semiciclo de 8,33 ms da rede elétrica, o TRIAC é acionado, após a deteç̧ão de cruzamento por zero, ilustrado pelo ponto 2 no eixo vertical da Figura 7.

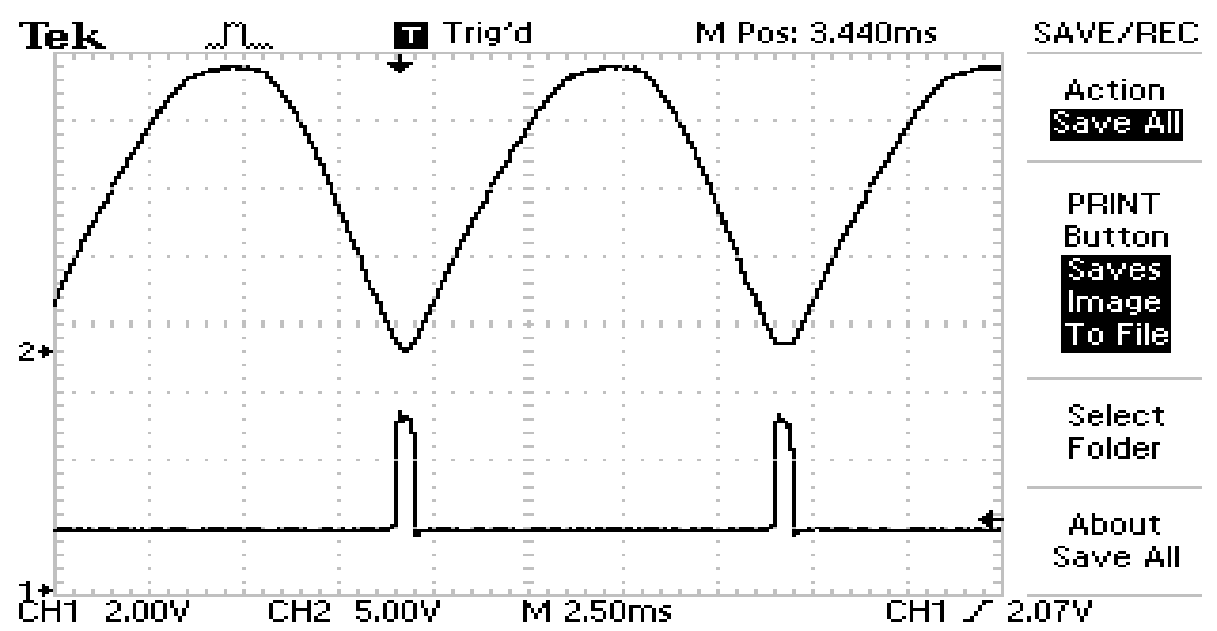

Figura 7: Tensão alternada rebatida da rede elétrica pela ponte de diodos e pulso de saída do circuito detector de cruzamento pelo zero.

Embora PWM seja muito utilizado para o controle de tensão contínua em aplicações de variação de cores com LEDs RGB, como também em motores de corrente contínua, foi adotado neste experimento um controle PWM para tensão alternada com largura de pulso de disparo variável, como ilustrado na Figura 8. Nesse caso, o período total do PWM é igual ao período do semiciclo (ps) da tensão senoidal da rede elétrica de aproximadamente $8,33 \mathrm{~ms}$, considerando a frequência da rede elétrica de $60 \mathrm{~Hz}$ e, consequentemente, cada ciclo completo correspondente a aproximadamente 16,6 ms.

Para o controle digital, este período do semiciclo de 8,33 ms foi dividido em 255 partes iguais para variação PWM, pois foi atribuido um byte (oito bits) de comunicação Bluetooth entre o aplicativo e o sistema microcontrolado para cada cor, ou seja, podendo ser transmitidos a variação de $2^{8}$ valores possíveis (de 0 a 255). Assim, cada parte unitária do período do semiciclo correspondeu a um intervalo de aproximadamente $33 \mu \mathrm{s}$.

Dessa forma, foi possível decidir em qual momento, de cada semicírculo da tensão senoidal da rede elétrica, será realizado o chaveamento pelo TRIAC e a condução da tensão eficaz para a carga Vca. Esse processo é conhecido também como controle do ângulo de fase ou momento de disparo do TRIAC. Assim, no controle do ângulo de fase, representado pela variação de ps, o TRIAC é acionado 
em um determinado instante, fazendo com que a carga esteja conectada à rede por um intervalo de tempo menor ou igual a um semicírculo (ALMEIDA, 2003).

Nesse caso, o erro máximo de potência entregue para a carga, devido à não-linearidade da tensão senoidal em relação à variação linear do ângulo de fase (ps), pode chegar a aproximadamente $10 \%$ (JUCÁ et al., 2013).

Neste experimento, o sinal de PWM foi atribuído ao pino de disparo do TRIAC (gate) e o início da contagem do PWM, indicado por ps, é determinado pelo circuito de zero-crossing que atribui zero ao valor de ps, através da interrupção externa do microcontrolador. Dessa forma, o valor ps é incrementado, a cada $32 \mu \mathrm{s}$, por interrupção do temporizador interno do microcontrolador, que compara o valor atual incrementado de ps e o valor enviado via Bluetooth pelo dispositivo Android. Caso o valor atual incrementado seja igual ao valor recebido anteriormente via Bluetooth, o sinal do PWM muda o estado de nível lógico baixo (valor digital "0") para nível lógico alto (valor digital "1"), disparando o TRIAC na borda de subida do PWM, em azul, como ilustrado na Figura 8.

Assim, o sinal do PWM é forçado a nível lógico baixo somente na próxima interrupção externa do microcontrolador ocasionada pelo zero-crossing, ou seja, após o período PWM de 8,33 ms, que corresponde ao período de cada semicírculo da rede.

Dessa forma, caso o valor de ps atribuído pelo dispositivo Android seja igual a zero, ocorre a comparação e o TRIAC é disparado no início do semicírculo fazendo com que a tensão senoidal seja fornecida integralmente à carga. Caso o valor de ps seja 128, ou seja, metade do período total de 255 partes de cada semiciclo, ocorre a condução pelo TRIAC de metade da onda da tensão senoidal para a carga.

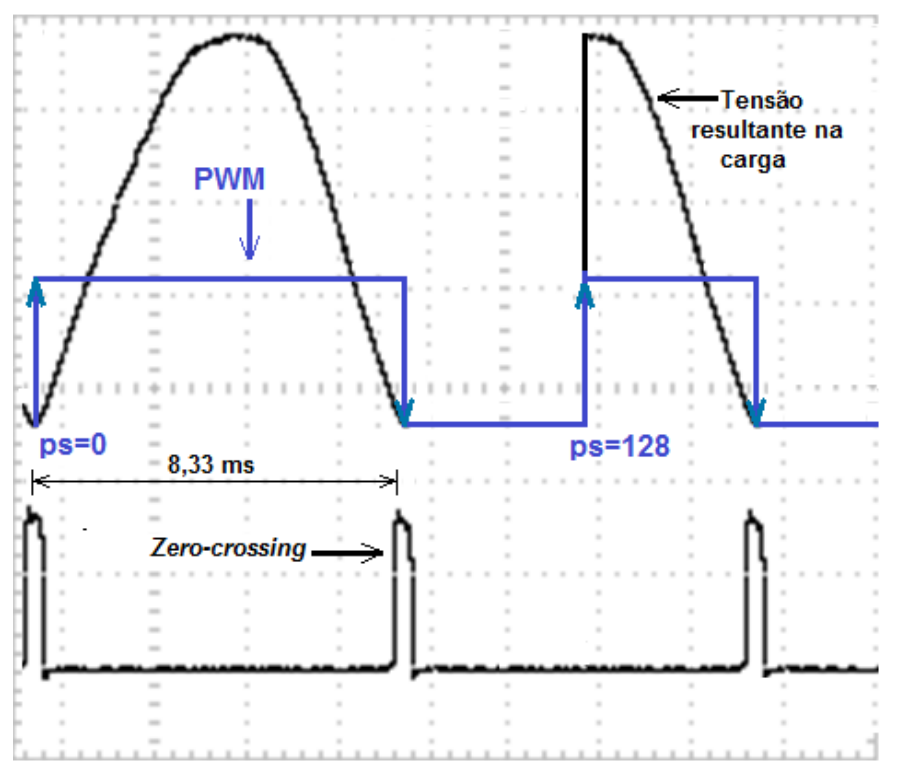

Figura 8: Ilustração de associação dos sinais de PWM, saída do circuito detector de cruzamento pelo zero e tensão senoidal resultante na carga conduzida pelo TRIAC.

As variações da tensão alternada da rede elétrica em práticas de eletricidade podem ser mostradas tanto com osciloscópios quanto através de lâmpadas incandescentes ou motores de corrente alternada como, por exemplo, de ventiladores domésticos. Neste experimento, ilustrado na Figura 9, foram aplicados 5\% em (a), 25\% em (b), 75\% em (c) e 100\% em (d) da tensão senoidal da rede elétrica em uma lâmpada incandescente através do controle digital por PWM. 


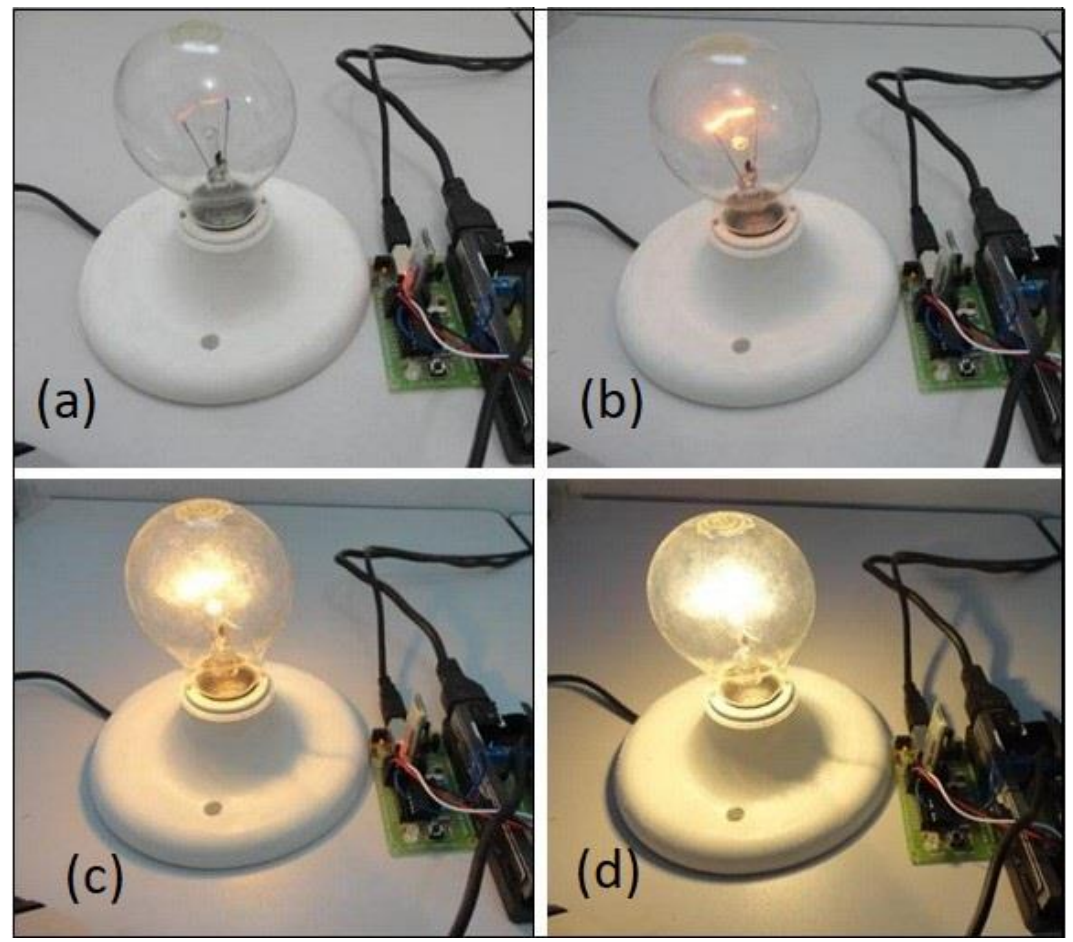

Figura 9: Aplicação do controle digital por PWM da tensão senoidal da rede elétrica em uma lâmpada incandescente.

O controle digital sem fio de tensão alternada, através do disco de graduação do software aplicativo Android desenvolvido, pode ser visualizado também pela variação de luminosidade de uma lâmpada incandescente em um ambiente escuro, como ilustra a Figura 10.
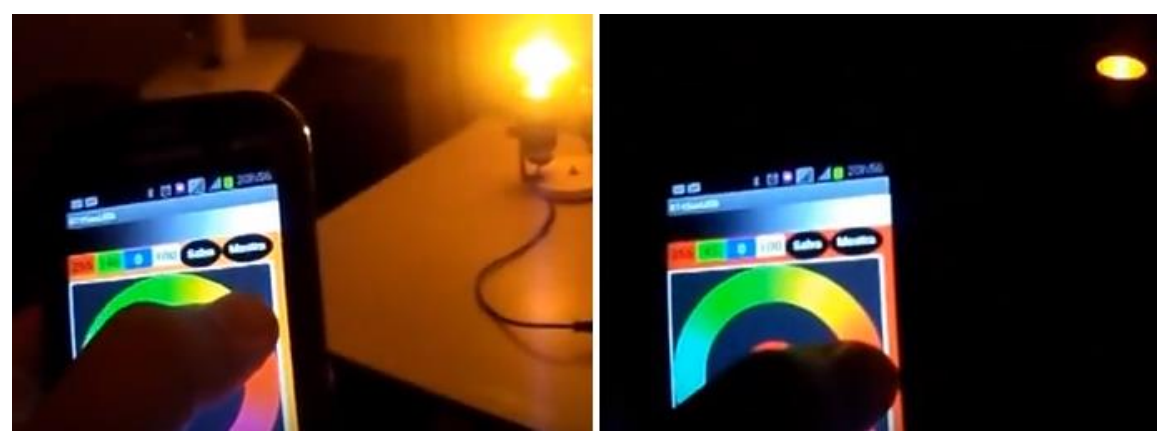

Figura 10: Controle digital sem fio de tensão alternada através do disco de graduação do software aplicativo desenvolvido utilizando dispositivo Android.

O programa do microcontrolador e o sketch para Arduino podem ser obtidos diretamente da tela do aplicativo BT4SanUSB.

O acionamento de cargas CA com controle digital possibilita também economia de energia, pois durante o controle digital, enquanto não ocorre o disparo do TRIAC, não há condução de corrente para a carga e, consequentemente, não há consumo de energia. Essa é uma das principais causas pela qual o controle digital passou a substituir o reostato original de motores.

\section{CONCLUSÃO}

Durante os experimentos de controle de tensão senoidal alternada, o sistema microcontrolado mostrou-se estável sem nenhuma oscilação na tensão gerada. O uso do Bluetooth possibilitou que 
diferentes perfis de usuários fossem capazes de instalar o aplicativo no dispositivo Android e controlálo diretamente através de emparelhamento com o módulo do sistema embarcado.

Os circuitos microcontrolados SanUSB e Arduino são open-source tanto em software, quanto em hardware, o que facilita o uso e disseminação destas ferramentas através de um grande número de bibliotecas e tutoriais disponíveis na Web.

Os softwares educacionais aplicativos desenvolvidos neste trabalho foram utilizados em práticas da disciplina de Eletricidade em cursos técnicos de Informática e em cursos superiores de Ciência da Computação do IFCE e, através dos relatos dos alunos após as práticas, foi verificado que essa ferramenta contribuiu de forma lúdica para a assimilação dos conteúdos abordado sobre eletricidade e tensão alternada. Além disso, esses softwares educacionais mostraram-se eficientes em relação à programação e também ao controle por comando de voz. Considerando que o custo de um dispositivo Android é bastante acessível, este projeto pode ser implementado em diversas instituições de ensino.

Todo o experimento de controle digital de tensão senoidal alternada foi baseado em software livre, visando o aprendizado e a interdisciplinaridade, em baixo custo, de física e informática.

\section{REFERÊNCIAS}

ALMEIDA, José Luiz Antunes de. Dispositivos Semicondutores: Tiristores. São Paulo: Érica, 2003. BRASIL, MEC. Secretaria de Educação Média e Tecnológica. Parâmetros Curriculares Nacionais: Ensino Médio. Part III. Ciências da Natureza, matemática e suas Tecnologias. Brasília: MEC/SEMTEC, 2000.

CAVALCANTE, Marisa Almeida; TAVOLARO, Cristiane Rodrigues Caetano.; MOLISANI, Elio. Física com Arduíno para iniciantes. Revista Brasileira de Ensino de Física, São Paulo, v. 33, n. 4, p. 4503, out./dez. 2011.

CAVALCANTE, Marisa Almeida. Novas Tecnologias no Estudo de Ondas Sonoras. Revista Brasileira de Ensino de Física, São Paulo, v. 30, n. 3, p. 579-613, dez. 2013.

DARROZ, Luiz Marcelo; DA ROSA, Cleci Terezinha Werner; DA ROSA, Álvaro Becker. Experimentos simples para visualização dos fenômenos de difração e interferência da luz. Revista Thema, Rio grande do sul, v. 13, n. 2, p. 18-26, 2016.

DE BONA, Aline; PARAVISI, Marcelo. O Whatsapp: um espaço de construção escolar e administrativa, Revista Thema, Rio grande do sul, v. 13, n. 1, p. 15-23, 2016.

JUCÁ, Sandro Cesar; CARVALHO, Paulo Cesar Marques de; PEREIRA, Renata Imaculada Soares; PETROV, Dmitri; HILLERINGMANN, Ulrich. Design and Implementation of a High Temperature Control Monitoring Applied to Micro Thermoelectric Generators, Renewable Energy and Power Quality Journal. v. 11, n. 1, 2013.

MULLER, Nathan. Bluetooth Demystified. New York: McGraw Hill, 2001.

OLIVEIRA, Gabriel Santos; SILVA, Alan Paranhos de Souza; PONTES, Danilo Leite; MARTINS, Maruedson Pires; DA SILVA, Robson Marinho. Análise Comparativa entre os kits proprietários e de robótica livre tendo como parâmetros o seu custo e características de ensino. XXXI Encontro Necional de Engenharia de Produção, Belo Horizonte, p. 2-9, out. 2011. 
ROCHA, Fábio Saraiva da; MARRANGHELLO, Guilherme Frederico; LUCCHESE, Márcia Maria. Acelerômetro eletrônico e a placa Arduino para ensino de Física em tempo real. Caderno Brasileiro de Ensino de Física, v. 31, n. 1, p. 124-148, 124 abr. 2014.

SOUZA, Anderson de; et al. A placa Arduino: uma opção de baixo custo para experiências de física assistidas pelo PC. Revista Brasileira de Ensino de Física, São Paulo, v. 33, n. 1, p. 1702, jan. 2011.

SANUSB. Ferramenta Computacional. Disponível em: http://sanusb.org/. Acesso em: 09 jun. 2018.

Submissão: 02/08/2018

Aceito: $29 / 09 / 2018$ 